\title{
Hyperactivité et fibromyalgie : la douleur en relais ? À propos d'un cas clinique
}

\author{
Hyperactivity and Fibromyalgia: Pain in Relay? About a Clinical Case
}

\author{
E. Bichet $\cdot$ A. Bioy \\ (C) Lavoisier SAS 2020
}

\begin{abstract}
Résumé Cet article met en avant la spécificité de l'expression douloureuse dans le syndrome fibromyalgique en lien avec les questionnements identitaires, en l'illustrant par une situation clinique, celle d'Émilie. La patiente s'est en effet structurée au prix d'une hyperactivité, permettant de pallier un équilibre psychique difficile. À la suite d'un accident de la voie publique, cette hyperactivité est empêchée, précipitant la patiente dans un risque majeur d'effondrement dépressif de décompensation d'une histoire de vie chaotique. C'est alors que la douleur chronique va venir prendre le relais afin de maintenir l'économie psychique du sujet, avec un fonctionnement presque analogue à ce qu'avait permis l'hyperactivité. Ce travail interroge l'apparition de la pathologie qui peut être envisagée comme un moment charnière dans le parcours de vie afin d'élaborer certaines angoisses jusque-là évitées.
\end{abstract}

Mots clés Fibromyalgie · Hyperactivité $\cdot$ États limites · Narcissique $\cdot$ Douleur

\begin{abstract}
This article highlights the specificity of painful expression in the fibromyalgia syndrome in connection with identity questioning, illustrated by a clinical situation, that of Emilie. The patient structured herself at the price of hyperactivity, allowing a difficult psychic balance to be overcome. Following an accident on the public highway, this hyperactivity is prevented, precipitating the patient into a major risk
\end{abstract}

\footnotetext{
E. Bichet $(\bowtie)$

Consultation pluridisciplinaire de la douleur, centre hospitalier de Châteauroux-Le Blanc, 216, avenue de Verdun, F-36000 Châteauroux, France e-mail : emeline-bichet@live.fr

A. Bioy $(\bowtie)$

Laboratoire de psychopathologie et neuropsychologie, université Paris-VIII, 2, rue de la Liberté,

F-93526 Saint-Denis cedex, France

e-mail : antoine.bioy@iedparis8.net
}

of depressive collapse and decompensation of a chaotic life history. It is then that chronic pain will take over in order to maintain the psychic economy of the subject, with a functioning similar to hyperactivity. This work questions the appearance of the pathology which can be considered as a pivotal moment in the life course in order to elaborate certain anxieties that have been avoided until now.

Keywords Fibromyalgia $\cdot$ Hyperactivity $\cdot$ Borderline Narcissistic · Pain

Dans les structures de prise en charge de la douleur chronique, nous rencontrons nombre de patients qui viennent en consultation avec un vécu douloureux intense et diffus assorti d'une demande fortement itérative et difficilement satisfaite, ou ceux pour lesquels il est complexe de parvenir à une accroche. Ceux-ci viennent mettre en difficulté les équipes par de puissants mouvements transférentiels ${ }^{1}$. Ces patients nous agitent, alimentent un vécu d'impuissance de chacun des acteurs avec une demande de « faire quelque chose » pour eux : un médicament, un conseil, une intervention chirurgicale... Cette demande d'agir et d'immédiateté vient court-circuiter une introspection pourtant nécessaire pour se réapproprier ce corps, souvent surmédicalisé et passif. C'est là qu'intervient le psychologue de la structure douleur, proposant une offre de mentaliser ce moment charnière de la douleur qui ankylose pensées et affects dans un fonctionnement opératoire tournant en boucle, voire à vide.

Plusieurs consultations, plusieurs échecs des thérapeutiques classiques, une impossibilité de poser un diagnostic traditionnel (rhumatismal, myotoxique, etc.), des examens

\footnotetext{
1 Le transfert désigne l'actualisation de modalités relationnelles antérieures à la situation lors d'une rencontre ayant lieu dans le présent. Ces mouvements affectifs « du passé » peuvent provenir du patient (transfert) ou du thérapeute à l'écoute de son patient (contretransfert). Lorsque le transfert n'est pas identifié, comme il renvoie à des situations passées, il peut mener à des impasses cliniques (par exemple, incompréhension mutuelle ne pas saisir ce qui « coince » dans la relation).
} 
complémentaires négatifs... « Rien n'est visible à la radio ou au scanner, rien ne se détecte aux examens de laboratoire actuels », décrit Sordet-Guepet [1]. Et c'est bien là le comble pour les patients : leur souffrance est invisible et inquantifiable, et échappe à l'evidence-based medicine. Il s'agit alors d'identifier et de nommer pour légitimer et reconnaître la souffrance du patient, et ainsi amorcer un travail thérapeutique.

\section{Émilie : l'accident comme une césure traumatique}

Dans le suivi des patients en structure douleur, nous observons régulièrement une impression de lutte constante et un sentiment d'impuissance. Le patient se débat avec son fonctionnement psychique et sa douleur «plus forts que lui ». À l'image d'Émilie, qui a 30 ans, un physique élancé et sportif, bien apprêtée tant par sa vêture que sa coiffure et son maquillage : elle est adressée en entretien psychologique par le médecin de la consultation douleur. Après un accident de la voie publique (AVP) sept mois plus tôt, les douleurs comme elle l'évoque «ne l'ont plus quittée ». Alors qu'initialement elle repart des services des urgences après l'AVP avec un diagnostic d'entorse cervicale, c'est celui de fibromyalgie qui sera finalement posé six mois après, les suites de l'accident ne justifiant plus ni l'intensité ni le caractère diffus des douleurs qui ont complètement enveloppé son corps. Pour Émilie, c'est l'accident qui va opérer comme une véritable rupture dans son parcours de vie, qui devient biphasique [2] : avant l'accident, elle était sur tous les fronts, dans un fonctionnement hyperactif dans toutes les sphères de sa vie : professionnelle, familiale, de loisirs, etc. Puis, « plus rien ». Auparavant, ce paradis hyperactif, opératoire, était dominé par le faire puis survient la douleur intraitable provoquée par un traumatisme physique bénin [2].

Émilie ne conduit pas lors de cet accident et est hospitalisée le temps de diagnostiquer et prendre en charge son entorse cervicale. Cependant, les éprouvés lors de l'accident sont intenses, sidérants, et relèvent de la symptomatologie du trouble de stress post-traumatique : Émilie fait part d'un réel effroi et évoque même une envie de se jeter sur la route directement après l'impact, avec une atteinte narcissique forte : «Je me répétais que j'étais nulle, là, sur cette route. J'avais le sentiment de n'être plus rien. Je n'existais pas. "» Le vécu d'effroi, de sidération, la confrontation au réel de la mort et son aspect effractant évoquent une véritable césure traumatique que la symptomatologie des semaines et mois suivants viendra confirmer.

Ce traumatisme vient attaquer de façon importante le fonctionnement hyperactif qu'Émilie avait mis en place jusque-là. Ainsi, depuis le début de ses études, dès qu'une difficulté se présentait, elle avait recours à un agir extrême, allant jusqu'à mettre à l'épreuve et attaquer son corps dans un mouvement masochiste parfois violent. Depuis l'accident, ce fonctionnement hyperactif se trouve parfois activé, mais semble ne plus réellement fonctionner. Émilie continue pourtant à $\mathrm{y}$ avoir recours dès que possible, la crainte majeure semble être celle de s'effondrer sous une forme dépressive, comme elle l'a déjà expérimenté en fin d'adolescence (Émilie fera d'ailleurs le lien entre cet épisode et son hyperactivité). Winnicott [3] a auparavant décrit les mouvements de réponse entre sidération face à un événement qui déborde, ici l'accident, et des vécus dépressifs plus précoces.

Après l'accident, Émilie ne peut plus travailler et associe la situation à un échec, voire un handicap. Ce d'autant plus qu'elle travaillait dans un lieu associant commerce et activité sportive. L'incapacité de poursuivre dans ce lieu de mouvement, d'endurance et d'efforts est doublement difficile : d'une part, car son hyperactivité n'a plus de lieux privilégiés d'expression et, d'autre part, car l'atteinte narcissique devient béante : elle ne peut plus être elle-même. Ce lieu était en effet celui d'une gratification importante (son expertise était appréciée des clients comme des collègues) recherchée ; une véritable quête narcissique : exister à travers cette reconnaissance, ce qui éloignait ses tensions internes sans toutefois les résoudre, ayant plus une fonction autocalmante $[4,5]$. C'est dans ce contexte que la douleur va prendre le relais à ce fonctionnement devenu insuffisant.

\section{L'hyperactivité : un moyen de défense à date limite}

Chez Émilie, l'hyperactivité est une modalité d'équilibre qui est apparue au sortir de l'adolescence. Plus que cela, elle est devenue une étiquette identitaire et, comme nous l'évoquions, un mode de satisfaction narcissique : "Avant je faisais ça... Et ça... et ça. Maintenant je ne fais plus ». Ce « faire » lui permet de s'éprouver par le corps, sans avoir recours au principe de symbolisation, qui est un élément de dynamisme et d'élaboration psychique [6] ouvrant vers la résolution de symptôme. Plus que cela, ce « faire » agissait comme des éléments de « contre-mesure » d'une dépression larvée depuis l'adolescence, qui a déjà connu un épisode majeur, mais qui depuis «n'a plus le droit d'être », comme le dit Émilie.

Depuis l'accident, ce moyen de défense contre l'effondrement dépressif s'érode et devient obsolète. Nous assistons alors à un repli sur les perceptions douloureuses sous la forme d'une plainte pour que soit fait quelque chose : une action doit venir là aussi procurer du soulagement, en même temps qu'Émilie n'a jamais réellement eu accès de sa vie à une forme de soulagement de sa tension interne. Jusqu'à présent, la mécanique était bien huilée en un tout cohérent, professionnellement et personnellement, avec des apports narcissiques en conséquence : sportive accomplie et professionnelle compétente dont l'hyperactivité était à la fois 
l'essence et le moteur de sa vie. Maintenant, elle remplace le merci qu'elle recevait de l'autre par la plainte qu'elle lui adresse. La douleur devrait avoir pour fonction de contenir ce que l'hyperactivité n'arrive plus à maintenir. Elle devient un faire, et un lieu où il faut faire des choses pour calmer. Dans la fibromyalgie d'Émilie, le corps est entièrement douloureux, parfois même « il n'y a pas un endroit où [elle n'a] pas mal » comme elle le verbalise, empêchant la fuite et l'angoisse que son moi se délite. Alors, la fibromyalgie ne pourrait-elle pas être considérée comme une mise en acte chez ces patients à l'organisation narcissique identitaire ? Un ultime recours à l'agir de façon analogue à l'hyperactivité, avec un corps entièrement douloureux : celui-ci viendrait comme tentative de mentalisation pour pallier un défaut de symbolisation.

\section{La fibromyalgie : une mise en acte douloureuse?}

Dès lors qu'un événement viendrait rompre cet équilibre économique, les défenses autrefois utilisées ne sont plus suffisantes et l'angoisse peut émerger. Autrement dit, les conflits et affects douloureux ne sont ni refoulés (névrose) ni contre-investis (hyperactivité), mais éjectés hors de la psyché par ce que l'on pourrait appeler actes symptômes.

Chez Émilie, le diagnostic de fibromyalgie tombe rapidement après la pérennisation des symptômes douloureux post-accident. Elle trouve un fondement biologique qui reste indéniable, avec une hypersensibilisation centrale et un seuil douloureux diminué après des années d'hyperactivité intense, durant lesquels les signaux d'alerte, de fatigue, voire d'épuisement n'étaient pas écoutés pour réguler son activité en conséquence. Elle se saisit de ce diagnostic qui légitime donc une douleur " qui n'avait plus lieu d'être » selon le corps médical, et s'en empare même de manière identitaire « je suis fibromyalgique », nous dira-t-elle, mais aussi par moments avec une certaine affectivité : « ma fibromyalgie», relatant l'ambivalence par rapport à ce diagnostic qui soulage, agresse et dont elle se saisit comme pour pallier le vide identitaire. Contrairement à ses proches, qu'elle a vécus comme la rejetant toute son enfance, les douleurs, elles, « ne la quittent plus » comme elle le verbalise. Clivée de sa vie affective, cela l'aurait défendue d'une rechute dans un fonctionnement dépressif tant redouté et déjà vécu. En outre, ce moment charnière de l'accident, mais aussi de la part diagnostique, permet de reconnaitre une souffrance qui n'avait jamais été reconnue.

Ces éléments sont rapidement remplacés par une répétition de la plainte de tout ce qu'il n'était plus possible de faire : il a fallu sortir de cette itération afin de pouvoir amorcer une conflictualisation de ce qu'Émilie avait pu traverser sans avoir l'impression « de le vivre ». Elle est stoppée par la maladie et peut commencer à évoquer son ambivalence : « il a bien fallu cela pour que je m'arrête et que je prenne conscience, que je m'occupe de moi», nous dit-elle.

Émilie ajoute après avoir pris conscience des «bénéfices » de cette pause forcée qu'elle « donnerait tout pour pouvoir revivre une journée speed $»:$ le paradis hyperactif reste idéalisé, et bien que le travail d'élaboration soit amorcé, il semblerait que ce fonctionnement soit au-delà, comme un procédé autocalmant [7] qui évoque aussi la question de la dépendance (elle y revient, comme un aspect qui lui serait nécessaire). Petit à petit, c'est donc la capacité d'être seule en présence de l'autre (ici le thérapeute) qui sera travaillée. La thérapeute devient donc le garant externe d'une autonomie subjective problématique et qu'il convient de réparer en s'occupant de soi d'abord ensemble, puis seule.

Alors que l'hyperactivité peut être considérée comme un contre-investissement dépressif, il semblerait que la chronicisation du syndrome douloureux pourrait constituer ici une mise en acte par le symptôme. Une énième mise en acte, qui s'observe dans le parcours d'Émilie quand l'enveloppe corporelle faillit à son tour et ne parvient plus à remplir sa fonction de procédé autocalmant : l'accident pourrait être perçu comme une occasion pour le corps de rendre compte de ce contrecoup et offrir un moment charnière propice à l'élaboration. C'est de ce « stop » dont parle Émilie, accident dans le réel mais aussi un accident symbolique dans sa vie, au sens propre de ce qui bouleverse et met en danger. Celle-ci peut s'entrevoir à travers la mise en danger de son narcissisme, de son Moi qui survivait tant bien que mal, contenu par son enveloppe de mouvement et d'activisme.

Dans ce contexte de rupture, d'effraction post-accident, la douleur pourrait venir remplir un rôle de relais, comme un Moi-peau secondaire [8] avec tous les apports qu'elle implique : la gratification auprès de ses proches, une fonction de reprise et de réflexivité afin de se sentir dans son corps, se redéfinir également par le biais de la reconnaissance du syndrome. Émilie a donc pu disposer de ce temps de remaniements forcé afin de mentaliser des éléments narcissiques essentiels, jusque-là en stase, comme la difficile question de l'identité mais aussi de la dépendance à l'autre, bien visible dans le cadre thérapeutique. Ainsi, Émilie " était quand elle bougeait », jusqu'à ce qu'elle ne puisse plus bouger. Dès lors, elle n'était plus et a dû penser ce fonctionnement pour arriver à ne pas s'effondrer psychiquement.

\section{Conclusion}

La question des diagnostics dans la douleur chronique comme celui de la fibromyalgie vient remettre en perspective nos compréhensions de ces syndromes douloureux qui peuvent être vecteurs d'expression de souffrances 
psychopathologiques. Il s'agit pour nous de pouvoir penser le sujet dans toute sa globalité, ce qui inclut un respect des dimensions de subjectivité et de temporalité propres à chacun. Plutôt que de considérer l'apparition de ces syndromes comme une désorganisation progressive, pourquoi ne pas les envisager comme une opportunité de vivre ces affects qui n'ont jamais eu l'occasion de pleinement l'être ? De ces éléments non seulement nous miserions sur l'attention au patient, mais aussi sur une prise en charge globale qui se doit d'intégrer la composante médicale et sociale, tout en permettant de pointer les ressources du patient et sa place dans son parcours de soin et, in fine, de vie.

Liens d'intérêts : Les auteurs déclarent n'avoir aucun lien d'intérêt.

\section{Références}

1. Sordet-Guepet H (2004) L'insaisissable fibromyalgie. Evol Psy 69:671-89

2. Burloux G (1999) Pourquoi la douleur ? RFP 63:43-55

3. Winnicott D (2001) Explorations conceptuelles. La crainte de l'effondrement et autres situations cliniques. Gallimard, Paris

4. Furlanetto A, Spoljar F (2017) De l'hyperactivité à la fibromyalgie : une dette insolvable (étude de cas). Cliniques méditerranéennes 2:19-35

5. Szwec G (1998) Les Galériens Volontaires - Essais sur les procédés autocalmants. PUF, Paris

6. Roussillon R (2014) Pertinence du concept de symbolisation primaire. In: Brun A, Roussillon R (eds) Formes primaires de symbolisation. Dunod, Paris, pp 155-74

7. Smadja C (1993) À propos des procédés autocalmants du Moi. Rev Fr Psychosom 4:9-26

8. Anzieu D (1995) Le Moi-Peau. Dunod, Paris 